\title{
Comparison of non-stent retriever and stent retriever mechanical thrombectomy devices for the endovascular treatment of acute ischemic stroke
}

\author{
Kate A. Hentschel, BS, ${ }^{1}$ Badih Daou, MD, ${ }^{1}$ Nohra Chalouhi, MD,, Robert M. Starke, MD, ${ }^{2}$ \\ Shannon Clark, MD, ${ }^{1}$ Ashish Gandhe, MD, ${ }^{3}$ Pascal Jabbour, MD, ${ }^{1}$ Robert Rosenwasser, MD, ${ }^{1}$ and \\ Stavropoula Tjoumakaris, MD'
}

\begin{abstract}
Departments of ${ }^{1}$ Neurosurgery and ${ }^{3}$ Radiology, Thomas Jefferson University, Philadelphia, Pennsylvania; and ${ }^{2}$ Department of Neurosurgery, University of Virginia Health System, Charlottesville, Virginia
\end{abstract}

\begin{abstract}
OBJECTIVE Mechanical thrombectomy is standard of care for the treatment of acute ischemic stroke. However, limited data are available from assessment of outcomes of FDA-approved devices. The objective of this study is to compare clinical outcomes, efficacy, and safety of non-stent retriever and stent retriever thrombectomy devices.

METHODS Between January 2008 and June 2014, 166 patients treated at Jefferson Hospital for Neuroscience for acute ischemic stroke with mechanical thrombectomy using Merci, Penumbra, Solitaire, or Trevo devices were retrospectively reviewed. Primary outcomes included 90-day modified Rankin Scale (mRS) score, recanalization rate (thrombolysis in cerebral infarction [ $\mathrm{TICl}$ score]), and incidence of symptomatic intracranial hemorrhages (ICHs). Univariate analysis and multivariate logistic regression determined predictors of mRS Score 3-6, mortality, and TICI Score 3.
\end{abstract}

RESULTS A total of 99 patients were treated with non-stent retriever devices (Merci and Penumbra) and 67 with stent retrievers (Solitaire and Trevo). Stent retrievers yielded lower 90-day NIH Stroke Scale scores and higher rates of 90-day $\mathrm{mRS}$ scores $\leq 2$ (22.54\% [non-stent retriever] vs $61.67 \%$ [stent retriever]; $p<0.001)$, TICI Score $2 b-3$ recanalization rates $(79.80 \%$ [non-stent retriever] vs $97.01 \%$ [stent retriever]; $p<0.001)$, percentage of parenchyma salvaged, and discharge rates to home/rehabilitation. The overall incidence of $\mathrm{ICH}$ was also significantly lower $(40.40 \%$ [non-stent retriever] vs $13.43 \%$ [stent retriever]; $p=0.002)$, with a trend toward lower 90 -day mortality. Use of non-stent retriever devices was an independent predictor of mRS Scores 3-6 ( $p=0.002)$, while use of stent retrievers was an independent predictor of TICI Score $3(p<0.001)$.

CONCLUSIONS Stent retriever mechanical thrombectomy devices achieve higher recanalization rates than non-stent retriever devices in acute ischemic stroke with improved clinical and radiographic outcomes and safety.

https://thejns.org/doi/abs/10.3171/2016.2.JNS152086

KEY WORDS endovascular procedures; thrombectomy; stroke; vascular disorders; interventional neurosurgery

$\mathrm{D}$ ESPITE recent cerebrovascular advances, ischemic stroke remains a leading cause of morbidity and mortality. The goal of acute ischemic stroke (AIS) interventions is to obtain arterial recanalization and cerebral reperfusion. Over the past decade, 4 mechanical thrombectomy devices have obtained US Food and Drug Administration approval for the treatment of AIS as the sole intervention or as an adjunct to intravenous tissue plasminogen activator (tPA).

The MERCI (Mechanical Embolus Removal in Cerebral Ischemia) and Penumbra Pivotal Stroke trials sug- gested that the non-stent retriever thrombectomy devices Merci Retriever (Stryker) and Penumbra System (Penumbra, Inc.) achieve higher rates of recanalization than medical thrombolytic therapy (intravenous tPA). ${ }^{5,17}$ The SWIFT (Solitaire with the Intention for Thrombectomy) and TREVO 2 (Thrombectomy Revascularization of Large Vessel Occlusions in Acute Ischemic Stroke) trials suggested that newer stent retrievers Solitaire FR (ev3 Neurovascular) and Trevo Pro Retriever (Stryker) have significantly higher rates of recanalization than Merci. ${ }^{13,16}$ Because these trials focused mainly on establishing safety

ABBREVIATIONS AIS = acute ischemic stroke; CTP = CT perfusion; DWI = diffusion-weighted imaging; ICH = intracranial hemorrhage; JHN = Jefferson Hospital for Neuroscience; $m R S=$ modified Rankin Scale; NIHSS = NIH Stroke Scale; $\mathrm{TICI}=$ thrombolysis in cerebral infarction; $\mathrm{tPA}=$ tissue plasminogen activator. SUBMITTED September 3, 2015. ACCEPTED February 11, 2016. 
and efficacy of the thrombectomy devices, the most recent 2013 American Heart Association guidelines for the management of AIS recommended that additional studies assess the efficacy of these devices in improving clinical outcomes..$^{10}$

The 2015 MR CLEAN (Multicenter Randomized Clinical Trial of Endovascular Treatment for Acute Ischemic Stroke), ESCAPE (Endovascular Treatment for Small Core and Anterior Circulation Proximal Occlusion with Emphasis on Minimizing CT to Recanalization Times), and EXTEND-IA (Extending the Time for Thrombolysis in Emergency Neurological Deficits-Intra-Arterial) trials all found improvements in clinical outcomes and recanalization without significant increases in mortality or symptomatic hemorrhage when endovascular treatment with thrombectomy devices was performed in comparison with the sole administration of intravenous tPA. $.^{2,48} \mathrm{How}-$ ever, these trials did not directly address the differences in outcomes among the 4 available mechanical thrombectomy devices. Our study compares clinical outcomes, efficacy, and safety of the non-stent retriever devices Merci Retriever and Penumbra System with those of the stent retrievers Solitaire FR and Trevo Pro Retriever in the endovascular treatment of AIS.

\section{Methods \\ Study Population}

Patients with acute ischemic stroke who underwent intraarterial mechanical thrombectomy at Jefferson Hospital for Neuroscience (JHN) from January 2008 through June 2014 were identified from a prospectively maintained database. Patients with preintervention arterial dissections were excluded. A total of 166 patients were included in this study.

\section{Patient Selection and Endovascular Procedure}

Patients suspected of AIS underwent noncontrast CT scanning, CT angiography, and CT perfusion (CTP) scans upon admission to Thomas Jefferson University Hospital. Patients with an NIH Stroke Scale (NIHSS) score $\geq 5$, ischemic penumbra, and an identifiable intracranial arterial thrombus were selected to receive mechanical thrombectomy. Merci was used from 2008 through 2014. The Penumbra 0.054-in reperfusion catheter and the 5MAX with separator were used from 2008 to 2012. Stent retrievers were introduced in 2011. During each year from January 2008 through June 2014, 15, 10, 20, 34, 40, 32, and 15 mechanical thrombectomies, respectively, were performed. Some patients were treated with multiple devices or were given supplemental intraarterial tPA. The first device used in each patient was the one reported for analysis. CT and MRI scans were obtained 24 hours postintervention to screen for hemorrhagic complications.

\section{Outcome Measures}

Primary outcome measures included modified Rankin Scale (mRS) score at 90 days, recanalization rate as assessed by the thrombolysis in cerebral infarction (TICI) score, ${ }^{9}$ and incidence of symptomatic intracranial hemorrhage (ICH). A good clinical outcome was defined as an $\mathrm{mRS}$ score $\leq 2$ at 90 days, while a poor outcome was de- fined as an mRS Score 3-6. Successful recanalization was defined as a TICI score of $2 b$ or 3. Symptomatic hemorrhages were identified as ICH associated with a worsening of the NIHSS by $\geq 4$ points or those requiring intervention.

Secondary outcomes included discharge location, mortality at 90 days, radiographic analysis of the percentage of area salvaged, and overall incidence of ICH. Discharge location was grouped by release to home or rehabilitation versus hospice, assisted living facility, or in-hospital death. An unbiased neuroradiologist blinded to the type of intervention patients received calculated percentage area of brain parenchyma salvaged in the 71 patients who underwent both preintervention CT scanning and postintervention MR imaging by dividing the area salvaged by initial area at risk. Radiographic findings ranging from petechiae to large-scale hemorrhages requiring intervention were included in the overall incidence of ICH.

\section{Statistical Analysis}

Data are presented as the mean and range for continuous variables and as frequency for categorical variables. Analysis was carried out using chi-square and Fisher's exact tests as appropriate. A comparison of continuous data was carried out using an unpaired t-test when there were 2 groups. When there were 3 or more groups, analysis was carried out using ANOVA followed by Bonferroni post hoc testing. Univariate analysis was used to test covariates predictive of the following dependent outcomes: $\mathrm{mRS}$ Score 3-6, mortality at 90 days, and TICI Score 3. Interaction and confounding were assessed through stratification and relevant expansion covariates. Factors predictive in univariate analysis $(p<0.20)$ were entered into a backward multivariate logistic regression analysis; $p$ values of $\leq 0.05$ were considered statistically significant. Statistical analysis was carried out using Stata (version 10.0, StataCorp.).

\section{Results \\ Baseline Characteristics}

Of the 166 patients with AIS who underwent mechanical thrombectomy at JHN, 99 were treated with non-stent retriever devices (30 treated with Merci and 69 treated with Penumbra) and 67 with stent retrievers (62 treated with Solitaire and 5 treated with Trevo). The non-stent retriever and stent retriever cohorts were statistically comparable with respect to age, sex, NIHSS score on admission, baseline $\mathrm{mRS}$, and occlusion site (Table 1). However, the stent retriever cohort included a significantly greater proportion of African Americans ( $\mathrm{p}=0.04)$. Additionally, a higher proportion of stent retriever patients had a history of smoking $(\mathrm{p}=0.01)$. Before initiating intraarterial mechanical thrombectomy, intravenous tPA was administered to 42 patients in the non-stent retriever cohort and 35 in the stent retriever group ( $\mathrm{p}=0.21)$. The average time from symptom onset to groin puncture was statistically comparable between the 2 cohorts.

\section{Clinical Outcomes}

Good clinical outcomes (mRS score $\leq 2$ at 90 days) were attained more often in the stent retriever cohort 
TABLE 1. Baseline characteristics of the study population

\begin{tabular}{|c|c|c|c|c|c|c|c|}
\hline Variable & $\begin{array}{l}\text { Non-Stent Retriever } \\
\qquad(\mathrm{n}=99)\end{array}$ & $\begin{array}{l}\text { Stent Retriever } \\
\qquad(n=67)\end{array}$ & p Value & $\operatorname{Merci}(n=30)$ & $\begin{array}{l}\text { Penumbra } \\
(n=69)\end{array}$ & $\begin{array}{l}\text { Stent Retriever } \\
\qquad(n=67)\end{array}$ & p Value* \\
\hline Mean age (SD), yrs & $66.17(14.66)$ & $62.66(16.50)$ & 0.15 & $66.36(13.66)$ & $66.09(15.16)$ & $62.66(16.50)$ & 0.36 \\
\hline Sex, $n(\%)$ & & & & & & & 0.94 \\
\hline Female & $53(53.54)$ & $34(50.75)$ & 0.72 & $16(53.33)$ & 37 (53.62) & $34(50.75)$ & \\
\hline Male & $46(46.46)$ & $33(49.25)$ & & $14(46.67)$ & $32(46.38)$ & $33(49.25)$ & \\
\hline \multicolumn{8}{|l|}{ Race, $n(\%)$} \\
\hline Caucasian & 76 (83.52) & $44(68.75)$ & $0.04 \dagger$ & $24(88.89)$ & $52(81.25)$ & $44(68.75)$ & \\
\hline African American & $9(9.89)$ & $17(26.56)$ & & $2(7.41)$ & $7(10.94)$ & $17(26.56)$ & \\
\hline Mean BMI (SD) & $28.81(7.15)$ & $29.25(5.93)$ & 0.68 & $29.56(7.88)$ & $28.50(6.85)$ & $29.25(5.93)$ & 0.71 \\
\hline Smoking, n (\%) & $26(28.57)$ & $32(47.76)$ & $0.01 \dagger$ & $10(38.46)$ & $16(24.62)$ & $32(47.76)$ & $0.02 \dagger$ \\
\hline Diabetes, n (\%) & $31(31.31)$ & $17(25.37)$ & 0.41 & $10(33.33)$ & $21(30.43)$ & $17(25.37)$ & 0.68 \\
\hline Hypertension, n (\%) & $85(85.86)$ & 55 (82.09) & 0.51 & 25 (83.33) & $60(86.96)$ & 55 (82.09) & 0.73 \\
\hline Hyperlipidemia, n (\%) & $63(63.64)$ & $51(76.12)$ & 0.09 & $18(60.00)$ & $45(65.22)$ & $51(76.12)$ & 0.21 \\
\hline Atrial fibrillation, $n(\%)$ & $39(39.39)$ & $26(38.81)$ & 0.94 & $8(26.67)$ & $31(44.93)$ & $26(38.81)$ & 0.23 \\
\hline CVA, $n(\%)$ & $13(13.13)$ & $11(16.42)$ & 0.56 & $3(10.00)$ & $10(14.49)$ & $11(16.42)$ & 0.71 \\
\hline CAD, n (\%) & $43(43.43)$ & $24(35.82)$ & 0.33 & $14(46.67)$ & $29(42.03)$ & $24(35.82)$ & 0.56 \\
\hline Mean admission NIHSS (SD) & $18.01(6.35)$ & $16.41(6.59)$ & 0.13 & & & & \\
\hline \multicolumn{8}{|l|}{ Baseline mRS score, $\mathrm{n}(\%)$} \\
\hline 0 & $94(98.95)$ & $65(98.48)$ & 0.65 & $28(100.00)$ & $66(98.51)$ & $65(98.48)$ & $>0.99$ \\
\hline 1 & $0(0.00)$ & $1(1.52)$ & & $0(0.00)$ & $0(0.00)$ & $1(1.52)$ & \\
\hline 2 & $1(1.05)$ & $0(0.00)$ & & $0(0.00)$ & $1(1.49)$ & $0(0.00)$ & \\
\hline$\geq 3$ & $0(0.00)$ & $0(0.00)$ & & $0(0.00)$ & $0(0.00)$ & $0(0.00)$ & \\
\hline \multicolumn{8}{|l|}{ Side of occlusion, $\mathrm{n}(\%)$} \\
\hline Right & $40(44.94)$ & $30(49.18)$ & 0.61 & $10(37.04)$ & $30(48.39)$ & $30(49.18)$ & 0.54 \\
\hline Left & $49(55.06)$ & $31(50.82)$ & & $17(62.96)$ & $32(51.61)$ & $31(50.82)$ & \\
\hline \multicolumn{8}{|l|}{ Site of occlusion, $\mathrm{n}(\%)$} \\
\hline ICA/ICAT & $30(30.30)$ & $17(25.37)$ & 0.79 & $6(20.00)$ & $24(34.78)$ & $17(25.37)$ & 0.58 \\
\hline MCA & $59(59.60)$ & $43(64.18)$ & & $21(70.00)$ & $3855.07)$ & $43(64.18)$ & \\
\hline Vertebral/basilar/ $\mathrm{P}_{1}$ & $10(10.10)$ & $7(10.45)$ & & $3(10.00)$ & 7 (10.14) & $7(10.45)$ & \\
\hline Received intravenous tPA, $\mathrm{n}(\%)$ & $42(42.42)$ & $35(52.24)$ & 0.21 & $11(36.67)$ & $31(44.93)$ & $35(52.24)$ & 0.35 \\
\hline $\begin{array}{l}\text { Mean symptom onset to groin } \\
\text { puncture, (SD), hrs }\end{array}$ & $7.08(5.02)$ & $6.92(3.96)$ & 0.82 & $6.71(4.22)$ & $7.24(5.35)$ & $6.91(3.96)$ & 0.85 \\
\hline
\end{tabular}

$\mathrm{BMI}=$ body mass index; $\mathrm{CAD}=$ coronary artery disease $; \mathrm{CVA}=$ cerebrovascular accident; ICA = internal carotid artery; ICAT = internal carotid artery terminus; $\mathrm{MCA}=$ middle cerebral artery.

* $p$ value applies to the 3-group comparison between the stent retrievers and the individual non-stent retrievers.

$\dagger$ Statistically significant.

(22.54\% [non-stent retriever] vs $61.67 \%$ [stent retriever]; $\mathrm{p}<0.001$ ), as illustrated in Table 2. The 90-day NIHSS score (4.71 [non-stent retriever] vs 2.49 [stent retriever]; $\mathrm{p}$ $=0.008)$ and the length of hospital stay were significantly reduced (12.36 days [non-stent retriever] vs 8.3 days [stent retriever], $\mathrm{p}=0.02$ ), while the percentage of patients discharged to home/rehabilitation was significantly greater in the stent retriever cohort $(61.62 \%$ [non-stent retriever] vs $80.60 \%$ [stent retriever]; $p=0.009)$. There was also a trend toward decreased mortality at 90 days (34.34\% [non-stent retriever] vs $20.90 \%$ [stent retriever]; $p=0.07$ ).

\section{Efficacy Outcomes}

Recanalization (TICI Scores $2 b-3$ ) was achieved in $79(79.80 \%)$ patients in the non-stent retriever cohort and $65(97.01 \%)$ in the stent retriever cohort $(\mathrm{p}<0.001)$, as shown in Table 3. Although time to recanalization was not statistically different (8.42 hours [non-stent retriever] vs 7.96 hours [stent retriever]; $p=0.53$ ), the mean number of device passes (2.64 [non-stent retriever] vs 2.23 [stent retriever]; $\mathrm{p}=0.03)$ and use of intraarterial tPA (35.71\% [non-stent retriever] vs $8.96 \%$ [stent retriever]; $\mathrm{p}<0.001$ ) were significantly reduced in the stent retriever group. The percentage of brain salvaged was also significantly greater in the stent retriever cohort (28.23\% [non-stent retriever] vs $62.30 \%$ [stent retriever]; $\mathrm{p}=0.002$ ).

\section{Safety Outcomes}

The combined incidence of postintervention hemorrhages was significantly reduced in the stent retriever cohort (40/99, 40.40\% [non-stent retriever] vs 9/67, 13.43\% [stent retriever]; $\mathrm{p}=0.002)$. Symptomatic ICH occurred 
TABLE 2. Comparison of clinical outcomes of devices

\begin{tabular}{|c|c|c|c|c|c|c|c|}
\hline Variable & $\begin{array}{c}\text { Non-Stent } \\
\text { Retrievers }(n=99)\end{array}$ & $\begin{array}{l}\text { Stent Retrievers } \\
\qquad(n=67)\end{array}$ & $p$ Value & $\begin{array}{c}\text { Merci } \\
(n=30)\end{array}$ & $\begin{array}{l}\text { Penumbra } \\
(n=69)\end{array}$ & $\begin{array}{l}\text { Stent Retrievers } \\
\qquad(n=67)\end{array}$ & p Value \\
\hline Mean length of stay (SD), days & $12.36(13.16)$ & $8.30(4.57)$ & $0.02^{*}$ & $14.2(18.74)$ & $11.57(9.88)$ & $8.30(4.57)$ & $0.03^{*}$ \\
\hline Discharge destination, $\mathrm{n}(\%)$ & & & $0.009^{*}$ & & & & \\
\hline Home, rehabilitation & $61(61.62)$ & $54(80.60)$ & & & & & \\
\hline Other† & $38(38.38)$ & $13(19.40)$ & & & & & \\
\hline Mean change in NIHSS score (SD) & $-7.97(6.20)$ & $-8.76(7.83)$ & 0.51 & & & & \\
\hline Mean NIHSS at 90 days, (SD) & $4.71(4.13)$ & $2.49(3.64)$ & $0.008^{*}$ & & & & \\
\hline mRS score at 90 days, $\mathrm{n}(\%)$ & & & $<0.001^{*}$ & & & & $0.001^{*}$ \\
\hline 0 & $2(2.82)$ & $14(23.33)$ & & $1(5.00)$ & $1(1.96)$ & $14(23.33)$ & \\
\hline 1 & $7(9.86)$ & $14(23.33)$ & & $5(25.00)$ & $2(3.92)$ & $14(23.33)$ & \\
\hline 2 & $7(9.86)$ & $9(15.00)$ & & $2(10.00)$ & $5(9.80)$ & $9(15.00)$ & \\
\hline 3 & $14(19.72)$ & $4(6.67)$ & & $1(5.00)$ & $13(25.49)$ & $4(6.67)$ & \\
\hline 4 & $4(5.63)$ & $4(6.67)$ & & $1(5.00)$ & $3(5.88)$ & $4(6.67)$ & \\
\hline 5 & $7(9.86)$ & $2(3.33)$ & & $2(10.00)$ & $5(9.80)$ & $2(3.33)$ & \\
\hline$\leq 2$ & $16(22.54)$ & $37(61.67)$ & & & & & \\
\hline Mortality at 90 days, $\mathrm{n}(\%)$ & $34(34.34)$ & $14(20.90)$ & 0.07 & $9(30.00)$ & $25(36.23)$ & $14(20.90)$ & 0.14 \\
\hline
\end{tabular}

* Statistically significant.

† Discharge destination "other" indicates hospice, assisted-living facility, or in-hospital death.

$\ddagger$ Change from baseline to discharge.

in 7 non-stent retriever patients $(7.07 \%)$ and only 1 stent retriever patient $(1.49 \%)(\mathrm{p}=0.15)$. Of those with postintervention hemorrhages, 37 non-stent retriever and 7 stent retriever patients had received heparin. There were no significant differences in the incidence of deep vein thrombosis $(9 / 99,9.09 \%$ [non-stent retriever] vs $2 / 67,2.99 \%$ [stent retriever]; $\mathrm{p}=0.20$ ), decompressive hemicraniectomy (6/99, 6.06\% [non-stent retriever] vs 4/67, 5.97\% SR; $\mathrm{p}>$ $0.99)$, or hydrocephalus $(4 / 99,4.04 \%$ [non-stent retriever] vs $0 / 67,0.00 \%$ [stent retriever]; $\mathrm{p}=0.15$ ).

\section{Predictors of mRS Score 3-6 at 90 Days}

Univariate logistic regression analysis of data identified 7 factors as predictors of mRS Score 3-6: increased age ( $p$ $<0.001$ ), less likely administration of intravenous tPA ( $p$ $=0.03)$, use of a first-generation device $(\mathrm{p}<0.001)$, longer length of stay $(p=0.02)$, decreased TICI score $(p<0.001)$, postintervention hemorrhage $(p=0.005)$, and less change in the area salvaged $(p=0.007)$. A multivariate analysis including preintervention characteristics, preintervention CTP, postintervention diffusion-weighted imaging (DWI), and change in area identified the use of non-stent retriever devices $(p=0.002)$, increased CTP area $(p=0.04)$, and less change in area of parenchymal involvement $(p=0.03)$ as the independent predictors of $\mathrm{mRS}$ Score 3-6, as indicated in Table 4. Use of a second-generation device rather than Merci was not a predictor of poor 90-day outcome (OR 0.53 [95\% CI 0.154-1.793], p = 0.30). However, use of Penumbra rather than Merci was a predictor of poor 90day outcome (OR 6.14 [95\% CI 1.436-26.218], $\mathrm{p}=0.01$ ).

\section{Predictors of Mortality at 90 Days}

Univariate logistic regression analysis of data identified 3 factors as predictors of mortality: increased age $(\mathrm{p}=0.02)$, history of diabetes $(\mathrm{p}=0.008)$, and decreased
TICI score $(p<0.001)$. A multivariate analysis including preintervention characteristics identified increasing age $(\mathrm{p}$ $=0.02)$ and history of diabetes $(\mathrm{p}=0.01)$ as independent predictors of mortality, as noted in Table 5. When controlling for these 2 factors, there was a trend toward decreased mortality with stent retrievers $(\mathrm{p}=0.07)$. Neither use of a stent retriever versus Merci (OR 0.697 [95\% CI $0.254-1.915], \mathrm{p}=0.48$ ) nor use of Penumbra versus Merci (OR 1.38 [CI 0.532-3.593], $\mathrm{p}=0.51$ ) was a predictor of mortality at 90 days.

\section{Predictors of TICI Score 3 Recanalization}

Univariate logistic regression analysis of data identified 3 factors as predictors of TICI Score 3 recanalization: use of a stent retriever $(\mathrm{p}<0.001)$, less likely administration of intraarterial tPA $(p=0.02)$, and decreasing number of device passes $(p=0.002)$. A multivariate analysis including preintervention characteristics identified the use of stent retrievers $(\mathrm{p}<0.001)$ as the only independent predictor of TICI Score 3, as specified in Table 6. Use of a stent retriever rather than Merci was a predictor of TICI Score 3 (OR 5.13 [CI 1.914-13.728], $\mathrm{p}=0.001$ ). However, use of Penumbra versus Merci was not a predictor (OR 1.01 [CI 0.409-2.503], $\mathrm{p}=0.98$ ).

\section{Discussion}

The goal of our analysis was to compare clinical outcomes, efficacy, and safety of 4 FDA-approved thrombectomy devices in the treatment of AIS. We hypothesized that stent retrievers (Solitaire and Trevo) would have better clinical outcomes, higher efficacy, and comparable safety in the treatment of AIS.

Our study found that mechanical thrombectomy using stent retrievers is associated with significantly higher rates of favorable clinical outcomes (mRS scores $\leq 2)$ in 
TABLE 3. Comparison of efficacy of devices

\begin{tabular}{|c|c|c|c|c|c|c|c|}
\hline Variable & $\begin{array}{c}\text { Non-Stent } \\
\text { Retrievers }(n=99)\end{array}$ & $\begin{array}{l}\text { Stent Retrievers } \\
\qquad(n=67)\end{array}$ & $\mathrm{p}$ Value & $\begin{array}{c}\text { Merci } \\
(n=30)\end{array}$ & $\begin{array}{l}\text { Penumbra } \\
(n=69)\end{array}$ & $\begin{array}{l}\text { Stent Retrievers } \\
\qquad(n=67)\end{array}$ & $\mathrm{p}$ Value \\
\hline \multicolumn{8}{|l|}{ Recanalization (TICl score), n (\%) } \\
\hline 1 & $12(12.12)$ & $1(1.49)$ & & $4(13.33)$ & $8(11.59)$ & $1(1.49)$ & \\
\hline 2 & $41(41.41)$ & $12(17.91)$ & & $14(46.67)$ & $27(39.13)$ & $12(17.91)$ & \\
\hline 3 & $38(38.38)$ & $53(79.10)$ & & $11(36.67)$ & $27(39.13)$ & $53(79.10)$ & \\
\hline \% salvaged (SD) & $28.23(54.33)$ & $62.3(33.77)$ & $0.002^{*}$ & & & & \\
\hline Mean time to recanalization, (SD), hrs & $8.42(5.11)$ & $7.96(4.08)$ & 0.53 & $8.07(4.11)$ & $8.58(5.51)$ & $7.96(4.08)$ & 0.73 \\
\hline Mean procedure duration, (SD), mins & $73.70(35.09)$ & $65.39(29.42)$ & 0.11 & & & & \\
\hline Intraarterial tPA, $n(\%)$ & $35(35.71)$ & $6(8.96)$ & $<0.001^{*}$ & $10(34.48)$ & $25(36.23)$ & $6(8.96)$ & $<0.001^{*}$ \\
\hline \multicolumn{8}{|l|}{ No. of device passes } \\
\hline
\end{tabular}

* Statistically significant.

TABLE 4. Factors predicting mRS Score $3-6$ at 90 days

\begin{tabular}{|c|c|c|c|c|}
\hline \multirow[b]{2}{*}{ Factor } & \multicolumn{2}{|c|}{ Univariate Analysis } & \multicolumn{2}{|c|}{ Multivariate Analysis } \\
\hline & OR $(95 \% \mathrm{Cl})$ & $p$ Value & OR $(95 \% \mathrm{Cl})$ & p Value \\
\hline Age & $1.04(1.019-1.071)$ & $<0.001^{*}$ & & \\
\hline Sex & $0.99(0.492-1.990)$ & 0.98 & & \\
\hline Race & $1.21(0.680-2.151)$ & 0.52 & & \\
\hline $\mathrm{BMI}$ & $1.03(0.976-1.081)$ & 0.31 & & \\
\hline Smoking & $0.96(0.469-1.978)$ & 0.92 & & \\
\hline Diabetes & $2.02(0.898-4.550)$ & 0.09 & & \\
\hline Hypertension & $2.56(0.967-6.783)$ & 0.06 & & \\
\hline Hyperlipidemia & $0.86(0.408-1.835)$ & 0.70 & & \\
\hline Atrial fibrillation & $1.94(0.907-4.141)$ & 0.09 & & \\
\hline CVA & $1.13(0.431-2.936)$ & 0.81 & & \\
\hline CAD & $1.47(0.708-3.064)$ & 0.30 & & \\
\hline Side of occlusion & $1.06(0.506-2.207)$ & 0.88 & & \\
\hline Intravenous tPA & $0.46(0.224-0.930)$ & $0.03^{*}$ & & \\
\hline Time to puncture & $0.95(0.878-1.025)$ & 0.18 & & \\
\hline Time to recanalization & $0.96(0.893-1.037)$ & 0.32 & & \\
\hline Device type & $0.18(0.084-0.387)$ & $<0.001^{*}$ & $0.09(0.021-0.405)$ & $0.002^{*}$ \\
\hline Intraarterial tPA & $0.72(0.316-1.635)$ & 0.43 & & \\
\hline No. of passes & $1.09(0.834-1.421)$ & 0.53 & & \\
\hline Length of stay & $1.08(1.015-1.159)$ & $0.02^{*}$ & & \\
\hline $\mathrm{TICl}$ score & $0.26(0.136-0.500)$ & $<0.001^{*}$ & & \\
\hline Hemorrhage & 3.77 (1.481-9.584) & $0.005^{*}$ & & \\
\hline DVT & $2.51(0.501-12.604)$ & 0.26 & & \\
\hline CTP area† & $1.00(0.990-1.001)$ & 0.09 & $1.02(1.001-1.046)$ & $0.04^{*}$ \\
\hline DWI areał & $1.01(0.997-1.021)$ & 0.13 & & \\
\hline Change in area§ & $0.99(0.982-0.997)$ & $0.007^{*}$ & $0.97(0.952-0.997)$ & $0.03^{*}$ \\
\hline $\begin{array}{l}\text { DVT = deep vein thrombosis. } \\
\text { * Statistically significant. } \\
\text { † Area of parenchymal involver } \\
\text { † Area of parenchymal involver } \\
\S \text { Change in area of parenchym }\end{array}$ & $\begin{array}{l}\text { intervention CTP. } \\
\text { tintervention DWI. } \\
\text { ent from pre- to postinte }\end{array}$ & & & \\
\hline
\end{tabular}


TABLE 5. Factors predicting mortality at 90 days

\begin{tabular}{|c|c|c|c|c|}
\hline \multirow[b]{2}{*}{ Factor } & \multicolumn{2}{|c|}{ Univariate Analysis } & \multicolumn{2}{|c|}{ Multivariate Analysis } \\
\hline & OR $(95 \% \mathrm{Cl})$ & $p$ Value & OR $(95 \% \mathrm{Cl})$ & $p$ Value \\
\hline Age & $1.03(1.006-1.056)$ & $0.02^{*}$ & $1.03(1.005-1.058)$ & $0.02^{*}$ \\
\hline Sex & $0.98(0.502-1.922)$ & 0.96 & & \\
\hline Race & $1.20(0.755-1.990)$ & 0.44 & & \\
\hline BMI & $1.03(0.977-1.078)$ & 0.31 & & \\
\hline Smoking & $1.58(0.792-3.132)$ & 0.20 & & \\
\hline Diabetes & $2.62(1.283-5.353)$ & $0.008^{*}$ & $2.59(1.251-5.347)$ & $0.01^{*}$ \\
\hline Hypertension & $3.44(0.978-12.084)$ & 0.05 & & \\
\hline Hyperlipidemia & $0.84(0.411-1.730)$ & 0.64 & & \\
\hline Atrial fibrillation & $1.26(0.639-2.492)$ & 0.50 & & \\
\hline CVA & $0.95(0.368-2.442)$ & 0.91 & & \\
\hline CAD & $1.22(0.617-2.402)$ & 0.57 & & \\
\hline Baseline mRS score & $1.21(0.188-7.814)$ & 0.84 & & \\
\hline Side of occlusion & $0.78(0.379-1.597)$ & 0.49 & & \\
\hline Intravenous tPA & $0.76(0.388-1.506)$ & 0.44 & & \\
\hline Time to puncture & $1.01(0.935-1.081)$ & 0.89 & & \\
\hline Time to recanalization & $1.00(0.936-1.079)$ & 0.89 & & \\
\hline Device type & $0.50(0.246-1.038)$ & 0.06 & $0.49(0.225-1.058)$ & 0.07 \\
\hline Length of stay & $0.98(0.945-1.023)$ & 0.41 & & \\
\hline No. of passes & $1.21(0.933-1.576)$ & 0.15 & & \\
\hline TICI Score 3 & $0.25(0.123-0.515)$ & $<0.001^{*}$ & & \\
\hline Hemorrhage & $1.99(0.929-4.251)$ & 0.08 & & \\
\hline Cerebral edema & $0.66(0.131-3.303)$ & 0.61 & & \\
\hline Decompressive hemicraniectomy & $1.61(0.431-6.044)$ & 0.48 & & \\
\hline Hydrocephalus & $1.21(0.107-13.685)$ & 0.88 & & \\
\hline
\end{tabular}

* Statistically significant.

TABLE 6. Factors predicting TICI Score 3 recanalization

\begin{tabular}{|c|c|c|c|c|}
\hline \multirow[b]{2}{*}{ Factor } & \multicolumn{2}{|c|}{ Univariate Analysis } & \multicolumn{2}{|c|}{ Multivariate Analysis OR $(95 \% \mathrm{Cl})$} \\
\hline & OR $(95 \% \mathrm{Cl})$ & $p$ Value & OR $(95 \% \mathrm{Cl})$ & p Value \\
\hline Age & $0.99(0.966-1.006)$ & 0.18 & & \\
\hline Sex & $0.93(0.507-1.724)$ & 0.83 & & \\
\hline Race & $1.16(0.730-1.837)$ & 0.53 & & \\
\hline BMI & $1.01(0.963-1.056)$ & 0.73 & & \\
\hline Smoking & $1.12(0.594-2.127)$ & 0.72 & & \\
\hline Diabetes & $0.96(0.491-1.890)$ & 0.91 & & \\
\hline Hypertension & $0.78(0.328-1.853)$ & 0.57 & & \\
\hline Hyperlipidemia & $1.57(0.809-3.048)$ & 0.18 & & \\
\hline Atrial fibrillation & $1.09(0.582-2.031)$ & 0.79 & & \\
\hline CVA & $1.92(0.780-4.746)$ & 0.16 & & \\
\hline CAD & $0.56(0.299-1.048)$ & 0.07 & & \\
\hline Side of occlusion & $1.08(0.563-2.054)$ & 0.83 & & \\
\hline Intravenous tPA & $1.31(0.711-2.432)$ & 0.38 & & \\
\hline Time to puncture & $0.96(0.898-1.029)$ & 0.25 & & \\
\hline Time to recanalization & $0.95(0.890-1.018)$ & 0.15 & & \\
\hline Device type & $6.08(2.973-12.420)$ & $<0.001^{*}$ & $6.08(2.973-12.420)$ & $<0.001^{*}$ \\
\hline Intraarterial tPA & $0.42(0.203-0.862)$ & $0.02^{*}$ & & \\
\hline Number of passes & $0.66(0.51-0.856)$ & $0.002^{*}$ & & \\
\hline
\end{tabular}

* Statistically significant. 
AIS patients, specifically $61.67 \%$ versus $22.54 \%$ for nonstent retriever devices. Similarly, the STAR (Solitaire FR Thrombectomy for Acute Revascularization) trial found a rate of good clinical outcomes of $57.9 \%$ with the use of Solitaire. ${ }^{14}$ The MERCI trial reported a rate of $\mathrm{mRS}$ score $\leq 2$ at 90 days of $27.7 \%$ with the use of the Merci Retriever. ${ }^{17}$ The SWIFT trial found that outcome is significantly improved with Solitaire (mRS score $\leq 2,58 \%$ ) as opposed to Merci (mRS score $\leq 2,33 \%) .{ }^{16}$ Ribo et al. and Deshaies et al. also documented greater rates of good clinical outcomes with the use of stent retrievers than Merci. ${ }^{7,15}$

The use of non-stent retriever devices $(\mathrm{p}=0.002)$, increased area of parenchymal involvement on preintervention CTP $(p=0.04)$, and less change in area of parenchymal involvement $(\mathrm{p}=0.03)$ were independent predictors of mRS Scores 3-6. Ribo et al. found that use of Solitaire and Trevo, compared with intraarterial tPA or Merci, increased the odds of an mRS score $\leq 2$ at 90 days (OR 1.9 [95\% CI 1.04-3.39], $\mathrm{p}=0.037) .{ }^{15}$ Kass-Hout et al. also reported that the use of stent retrievers was an independent predictor of good functional outcome (OR 2.27 [95\% CI 1.018-5.048], $\mathrm{p}=0.045) .{ }^{11}$ Greater preintervention parenchymal involvement is associated with higher risk of hemorrhagic conversion, which can explain its association with worse functional outcomes. ${ }^{18}$

Stent retrievers were associated with lower NIHSS score at 90 days $(\mathrm{p}=0.008)$ despite comparable scores upon admission. However, it is important to note that the trend toward lower initial NIHSS in the stent retriever group, although not statistically significant, may have been clinically relevant. Patients treated with stent retrievers had shorter hospital stays ( 8.3 days, $p=0.02)$ and higher discharge rates to home or rehabilitation $(80.60 \%, \mathrm{p}=$ $0.009)$. When controlling for increasing age and diabetes, the independent predictors of mortality, our study showed a trend toward decreased mortality with stent retrievers ( $p$ $=0.07$ ). Other studies also reported age as a significant predictor of mortality. ${ }^{1,12}$ Linfante et al. found diabetes to be a predictor of poor outcome, possibly due to the greater prevalence of diabetes among older patients who have higher odds of mortality after thrombectomy. ${ }^{12}$

Thrombectomy with stent retrievers yielded rates of recanalization (TICI Scores 2b-3) as high as $97.01 \%$. The EXTEND-IA trial and Dávalos et al. reported similar rates of TICI 2-3 recanalization. ${ }^{4,6}$ The SWIFT and TREVO 2 trials concluded that Solitaire and Trevo devices achieve significantly better recanalization grades than Merci. ${ }^{13,16}$ Ribo et al. also noted that Solitaire and Trevo (67.2\%) have higher recanalization rates than Merci $(57.3 \%)(\mathrm{p}=$ 0.050)..$^{5}$ The trend toward more intravenous tPA in the stent retriever group, as well as the greater number of occlusions at the terminus of the internal carotid artery and fewer middle cerebral artery occlusions, although not statistically significant, may have been clinically relevant. Our study found that the use of stent retrievers was the only independent predictor of TICI Score 3 recanalization $(\mathrm{p}<0.001)$. Decreased TICI score was a predictor of mortality, suggesting the significance of achieving recanalization on clinical outcomes.

The percentage of brain parenchyma salvaged increased significantly with the use of stent retrievers $(62.30 \%$ vs $28.23 \%, \mathrm{p}=0.002)$. Improved radiographic outcomes were consistent with greater recanalization. Additionally, the significantly lower use of intraarterial tPA $(p<0.001)$ and fewer device passes $(p=0.03)$ to achieve recanalization in the stent retriever cohort support the superior efficacy of stent retrievers. Minimizing the use of intraarterial tPA and device passes may offer additional benefits beyond recanalization by reducing the risk of hemorrhagic complications from thrombolysis or mechanical disruption of the arterial wall.

The incidence of postprocedural symptomatic ICH did not differ significantly between our non-stent retriever and stent retriever cohorts $(\mathrm{p}=0.15)$, while the total occurrence of hemorrhages was significantly lower with stent retrievers $(p=0.002)$. This might be attributed to more technical difficulties encountered with non-stent retrievers, potentially leading to a greater risk for vessel perforation associated with small insignificant or asymptomatic hemorrhages, or to the greater use of heparin prior to non-stent retriever procedures. Similarly, the SWIFT trial reported that the incidence of symptomatic ICH was nonsignificantly lower in patients treated with Solitaire, but the overall symptomatic and asymptomatic ICH incidence was significantly higher with Merci. ${ }^{16}$ Ribo et al. also found comparable rates of symptomatic ICH between the Solitaire and Merci devices $(\mathrm{p}=0.89) \cdot{ }^{15}$ In our study, the decrease in total occurrence of hemorrhages in association with comparable rates of symptomatic hemorrhages, decompressive hemicraniectomy, hydrocephalus, and deep venous thrombosis suggest improved safety with stent retrievers.

The primary limitation of this study is its retrospective nature. Although this was not a randomized blinded study, the collection database is prospectively maintained and the similarities in baseline characteristics between the 2 cohorts suggest that the results are valid. The limited sample size required some grouped analysis, rather than analysis of each device individually. Our study also lacked a control group receiving only intravenous tPA. However, our favorable results strongly support the use of stent retriever mechanical thrombectomy devices, either alone or as an adjunct to intravenous tPA, in the treatment of AIS. The revascularization rates achieved in our study exceed those attained from the sole use of intravenous tPA in published literature, such as the IMS III (Interventional Management of Stroke III) trial with an intravenous tPA revascularization rate of $40.8 \% .^{3}$ Additionally, the frequency of mechanical thrombectomy at JHN increased from 2008 through June 2014, which may reflect changes in stroke management over time. Finally, the results of this study relate to mechanical thrombectomy performed at a single institution and, thus, may not be applicable to other institutions.

\section{Conclusions}

Our study suggests that mechanical thrombectomy with stent retrievers results in favorable clinical and radiographic outcomes, high rates of recanalization, and improved safety in patients with AIS. These findings favor the use of stent retrievers (Solitaire and Trevo) over nonstent retriever devices (Merci and Penumbra) as first-line intraarterial treatment of intracranial occlusions. Addi- 
tional multiinstitutional studies should be done to confirm our findings.

\section{Acknowledgments}

This study was supported by the Sidney Kimmel Medical College Student Summer Research Program (to Ms. Hentschel).

\section{References}

1. Almekhlafi MA, Davalos A, Bonafe A, Chapot R, Gralla J, Pereira VM, et al: Impact of age and baseline NIHSS scores on clinical outcomes in the mechanical thrombectomy using solitaire FR in acute ischemic stroke study. AJNR Am J Neuroradiol 35:1337-1340, 2014

2. Berkhemer OA, Fransen PS, Beumer D, van den Berg LA, Lingsma HF, Yoo AJ, et al: A randomized trial of intraarterial treatment for acute ischemic stroke. N Engl J Med 372:11-20, 2015

3. Broderick JP, Palesch YY, Demchuk AM, Yeatts SD, Khatri P, Hill MD, et al: Endovascular therapy after intravenous t-PA versus t-PA alone for stroke. N Engl J Med 368:893903, 2013

4. Campbell BC, Mitchell PJ, Kleinig TJ, Dewey HM, Churilov L, Yassi N, et al: Endovascular therapy for ischemic stroke with perfusion-imaging selection. N Engl J Med 372:10091018, 2015

5. Clark W, Lutsep H, Barnwell S, Nesbit G, Egan R, North E, et al: The penumbra pivotal stroke trial: safety and effectiveness of a new generation of mechanical devices for clot removal in intracranial large vessel occlusive disease. Stroke 40:2761-2768, 2009

6. Dávalos A, Pereira VM, Chapot R, Bonafé A, Andersson T, Gralla J: Retrospective multicenter study of Solitaire FR for revascularization in the treatment of acute ischemic stroke. Stroke 43:2699-2705, 2012

7. Deshaies EM, Singla A, Villwock MR, Padalino DJ, Sharma S, Swarnkar A: Early experience with stent retrievers and comparison with previous-generation mechanical thrombectomy devices for acute ischemic stroke. J Neurosurg 121:12-17, 2014

8. Goyal M, Demchuk AM, Menon BK, Eesa M, Rempel JL, Thornton J, et al: Randomized assessment of rapid endovascular treatment of ischemic stroke. N Engl J Med 372:10191030,2015

9. Higashida RT, Furlan AJ, Roberts H, Tomsick T, Connors B, Barr J, et al: Trial design and reporting standards for intra-arterial cerebral thrombolysis for acute ischemic stroke. Stroke 34:e109-e137, 2003

10. Jauch EC, Saver JL, Adams HP Jr, Bruno A, Connors JJ, Demaerschalk BM, et al: Guidelines for the early management of patients with acute ischemic stroke: a guideline for healthcare professionals from the American Heart Association/ American Stroke Association. Stroke 44:870-947, 2013

11. Kass-Hout T, Kass-Hout O, Sun CH, Kass-Hout T: Clinical, angiographic and radiographic outcome differences among mechanical thrombectomy devices: initial experience of a large-volume center. J Neurointerv Surg 7:176-181, 2015

12. Linfante I, Starosciak AK, Walker GR, Dabus G, Caston- guay AC, Gupta R, et al: Predictors of poor outcome despite recanalization: a multiple regression analysis of the NASA registry. J Neurointerv Surg 8:224-229, 2016

13. Nogueira RG, Lutsep HL, Gupta R, Jovin TG, Albers GW, Walker GA, et al: Trevo versus Merci retrievers for thrombectomy revascularisation of large vessel occlusions in acute ischaemic stroke (TREVO 2): a randomised trial. Lancet 380:1231-1240, 2012

14. Pereira VM, Gralla J, Davalos A, Bonafé A, Castaño C, Chapot R, et al: Prospective, multicenter, single-arm study of mechanical thrombectomy using Solitaire Flow Restoration in acute ischemic stroke. Stroke 44:2802-2807, 2013 (Erratum in Stroke 44:e239, 2013)

15. Ribo M, Molina CA, Jankowitz B, Tomasello A, Zaidi S, Jumaa M, et al: Stentrievers versus other endovascular treatment methods for acute stroke: comparison of procedural results and their relationship to outcomes. J Neurointerv Surg 6:265-269, 2014

16. Saver JL, Jahan R, Levy EI, Jovin TG, Baxter B, Nogueira RG, et al: Solitaire flow restoration device versus the Merci Retriever in patients with acute ischaemic stroke (SWIFT): a randomised, parallel-group, non-inferiority trial. Lancet 380:1241-1249, 2012

17. Smith WS, Sung G, Starkman S, Saver JL, Kidwell CS, Gobin YP, et al: Safety and efficacy of mechanical embolectomy in acute ischemic stroke: results of the MERCI trial. Stroke (Phila Pa 1976) 36:1432-1438, 2005

18. Souza LC, Payabvash S, Wang Y, Kamalian S, Schaefer P, Gonzalez RG, et al: Admission CT perfusion is an independent predictor of hemorrhagic transformation in acute stroke with similar accuracy to DWI. Cerebrovasc Dis 33:8-15, 2012

\section{Disclosures}

Dr. Tjoumakaris reports that he is a consultant for Stryker and Covidien.

\section{Author Contributions}

Conception and design: Tjoumakaris, Hentschel, Daou, Chalouhi, Clark, Jabbour, Rosenwasser. Acquisition of data: Hentschel, Starke, Clark, Gandhe. Analysis and interpretation of data: Tjoumakaris, Hentschel, Daou, Chalouhi, Starke, Clark, Gandhe. Drafting the article: Tjoumakaris, Hentschel, Daou, Chalouhi. Critically revising the article: Tjoumakaris, Daou, Chalouhi, Clark. Reviewed submitted version of manuscript: Tjoumakaris, Hentschel, Daou, Clark, Gandhe, Jabbour, Rosenwasser. Approved the final version of the manuscript on behalf of all authors: Tjoumakaris. Statistical analysis: Starke. Administrative/ technical/material support: Rosenwasser. Study supervision: Tjoumakaris, Chalouhi, Jabbour, Rosenwasser.

\section{Correspondence}

Stavropoula Tjoumakaris, Division of Neurovascular Surgery and Endovascular Neurosurgery, Department of Neurological Surgery, Thomas Jefferson University Hospital, 909 Walnut St., 2nd Fl., Philadelphia, PA 19107. email: stavropoula. tjoumakaris@jefferson.edu. 